MITOCHONDRIAL DNA Symmetric Transcription

from our Cell Biology Correspondent

THE transcription of double helical DNA by DNA dependent RNA polymerase is generally assumed to be an asymmetric process with only one of the two strands of the DNA, the coding strand, being transcribed into messenger or one of the stable species of RNA. In Escherichia coli, as Travers, Bautz and Burgess and their associates have shown, the ability of RNA polymerase to select and transcribe the coding strand depends on the presence of sigma factor, without which transcription is symmetric.

Mitochondria which seem to have evolved from symbiotic bacteria, not only contain small double stranded and closed circular DNA molecules but also their own RNA polymerase, which is distinct from that in the nucleus of eukaryotic cells. As Küntzel and Schäfer reported recently (Nature New Biology, 231, 265 ; 1971), the RNA polymerase in the mitochondria of Neurospora crassa is a much simpler and smaller molecule than $E$. coli RNA polymerase ; it comprises a single polypeptide chain with a molecular weight of about 64,000 . On reflexion therefore, Aloni and Attardi's report (Proc. US Nat. Acad. Sci., 68, 1751; 1971) that both the strands of the mitochondrial DNA of HeLa cells are transcribed in vitro is perhaps not all that surprising. Symmetric transcription may perhaps be the evolutionary price mitochondria have had to pay for their simple genome and its RNA polymerase.

The two strands of HeLa cell mitochondrial DNA can be separated by appropriate centrifugation, and Attardi and his colleagues have developed methods for isolating mitochondrial RNA made in vivo after pulse labelling with uridine. Determining whether transcription is symmetric or asymmetric can therefore be determined by comparatively straightforward DNA. RNA hybridization experiments. Aloni and Attardi find that RNA made in the mitochondria during short (1-5 min) pulses hybridizes equally to the heavy (H) and light (L) strands, but RNA made during longer pulses hybridizes preferentially with the $\mathrm{H}$ strand. They have already obtained evidence which indicates that the entire $\mathrm{H}$ strand is transcribed, and this together with the finding that RNA made during short pulses hybridizes equally with $\mathrm{H}$ and $\mathrm{L}$ strands, and the rapid sedimentation of the L strand RNA, suggests that like the $H$ strand the $L$ strand is extensively and perhaps completely transcribed. The $\mathrm{L}$ strand transcript, however, rapidly disappears from the mitochondria either because it is degraded or possibly because it is exported to the cell cytoplasm. Why the transcription of mitochondrial
DNA is an exception to the rule of asymmetric transcription remains to be seen.

Schäfer and Küntzel and their colleagues (Europ. J. Biochem., 21, 478 ; 1971), having isolated the RNA polymerase of $N$. crassa mitochondria, have now devised a method for isolating intact the DNA from these mitochondria. They find it is a circular DNA some $25 \mu \mathrm{m}$ long, in other words some five times longer than the mitochondrial DNA of HeLa and other vertebrate cells; this leads them to speculate that the mitochondria in fungi may represent an early stage in the reductive evolution of bacterial endosymbionts which culminated in the mitochondria of vertebrates with their very small genomes. Using mitochondrial DNA from $N$. crassa, Schäfer et al. have investigated its transcription in vitro by $E$. coli RNA polymerase with sigma factor and conclude that there are about eight to nine specific binding sites for this mitochondrial genome. Some 50 per cent of the RNA transcribed in vitro by the bacterial enzyme seems from hydridization experiments to be ribosomal and transfer RNA. Among the remainder of the in vitro product are RNA strands which self anneal and may well be the product of symmetrical transcription of regions of the genome other than those which specify the stable mitochondrial RNAs. As Schäfer and Küntzel have themselves shown, however, the mitochondrial RNA polymerase is very different from the bacterial enzyme and it would be interesting to see what RNAs are transcribed in vitro by the mitochondrial enzyme.

\section{. \\ Response to Humidity}

from a Correspondent

TRANSPIRATION in plants is controlled by adjustments in the size of minute pores, the stomata, which perforate the surface of leaves. Each stoma consists of a slit bounded by two specialized epidermal cells, or guard cells, which determine its size. When the guard cells take up water and swell their shape alters in such a way that the pore opens and when they become less turgid it closes. Stomata are normally in an open or partly-open condition during the day and closed at night. As a result, diffusion of carbon dioxide into a leaf, which follows the same pathway as

\title{
Seismic Signals from Underground Explosions
}

IN next Monday's Nature Physical Science, A. Douglas, P. D. Marshall and D. J. Corbishley, from UKAEA, Blacknest, suggest reasons why some of the seismic signals from underground explosions are more complex than others. The basis of their idea is that the direct $P$ waves recorded in a complex signal have passed through material with a lower average value of the quality factor, $Q$, and have been alternated.

Most $\mathbf{P}$ signals from underground explosions are simple in character and usually comprise a single pulse--two to three cycles long-followed by a tail of lower amplitude arrivals which can be explained in terms of reflexions in the crust or upper mantle. But the less common complex signals have a tail with an amplitude that is similar to or even larger than the first arrival.

Douglas et al. point out that, according to their theory, the tails of complex signals should contain more high frequency energy than the direct $\mathbf{P}$ waves because high frequencies are selectively attenuated in materials of low $Q$; complex signals from an underground explosion should therefore point to lower average explosion magnitudes than the simple signals because of the reduction of the amplitude of the first arrivals. To test the whole theory Douglas et al. have examined the $\mathbf{P}$ seismograms of two underground explosions-one fired near Bukhara in the Soviet Union and the other (the nuclear explosion Longshot detonated on October 29, 1965) at Amchitka in the Aleutian Islands.

The vibrations from the Russian explosion were detected at Eskdalemuir in Scotland and at Gauribidanur in India, and the records from these two stations provide good examples of simple and complex signals from the same source. The record at Gauribidanur is complex and there is clear evidence of late arrivals with a higher dominant frequency than the earlier arrivals. And the magnitude of the explosion as measured from the Eskdalemuir records is higher than that indicated by the Gauribidanur data, suggesting that the direct $P$ waves arriving at Gauribidanur had been attentuated.

Douglas et al. detail two examples of complex signals from the Longshot explosion (both recorded in British Columbia) from which the magnitude of the explosion was estimated to be 4.8 and 5.4 ; this is much lower than the average estimate of 6.2 from the data of the arrays operated by the United Kingdom Atomic Energy Authority which all detected a simple signal. They also find that the beginnings of the complex signals have a dominant frequency of $\sim 0.8 \mathrm{~Hz}$ compared with a dominant tail frequency of $\sim 1.4 \mathrm{~Hz}$, again in accord with the theoretical predictions. 